\title{
Análisis clínico y microbiológico de la sepsis grave y el choque séptico por Escherichia coli en Medellín, Colombia
}

\author{
Camilo A. Restrepo-Álvarez', Elisa Bernal', Johana Ascuntar-Tello y Fabián Jaimes ${ }^{1,2}$
}

Severe sepsis and septic shock by Escherichia coli, clinical and microbiological analysis in Medellin, Colombia

Background: Escherichia coli is a common cause of a broad spectrum of infections, from non-complicated urinary tract infection, to severe sepsis and septic shock, that are associated to high impact outcomes, such as ICU admission and mortality. Aims: To establish differences in mortality, ICU admission, ESBL positive strains and antibiotic treatment, between patients with $E$. coli related severe sepsis and septic shock, with or without bacteremia and its variability based on the source of infection. Method: Secondary data analysis of a multicentric prospective cohort study. Results: From 458 patients with $E$. coli isolation, 123 had E. coli exclusively in blood culture, 222 solely in urine culture, and 113 in both samples. Escherichia coli isolation exclusively in blood culture was associated with higher frequency of ICU admission $(n=63 ; 51.2 \%)$, higher rate of mechanical ventilation requirement $(\mathrm{n}=19 ; 15.5 \%)$, higher mortality and longer hospital stay $(\mathrm{n}=22 ; 18 \%$; median of 12 days, IQR $=7$ -17 , respectively); but with a lower occurrence of ESBL strains, compared to patients with positive urine culture and positive blood/urine cultures $(n=20 ; 17.7 \%$ and $n=46 ; 20.7 \%$, respectively). 424 patients $(92.6 \%)$ received antibiotic treatment in the first 24 hours. The most commonly prescribed was piperacilin/tazobactam $(\mathrm{n}=256$; $60.3 \%$ ). The proportion of patients empirically treated with carbapenems vs non-carbapenems was similar in the three groups. Discussion: The source of infection, associated with ESBL strains risk factors, are useful tools to define prognosis and treatment in this population, because of their clinical and microbiological variability. Conclusion: Patients with $E$. coli isolation exclusively in the blood culture had higher frequency of non-favorable outcomes in comparison to patients with $E$. coli in urine culture with or without bacteremia. Additionally, in our population patients with E. coli solely in blood culture have lower prevalence of ESBL positive strains.

Keywords: Sepsis; septic shock; urinary tract infections; mortality; Escherichia coli.

Palabras clave: Sepsis; choque séptico; infección urinaria; mortalidad; Escherichia coli.

\section{Introducción}

$E$ scherichia coli, perteneciente a la familia Enterobacteriaceae, es reconocida como una causa importante de infección urinaria, sepsis, bacteriemia, meningitis neonatal e infección gastrointestinal ${ }^{1}$. Aunque los microorganismos grampositivos han ido aumentando como causa de sepsis debido a la instrumentación de la atención médica, entendida como la utilización de dispositivos o herramientas de manera invasora para el tratamiento o diagnóstico de los pacientes, y a las infecciones asociadas al cuidado de la salud ${ }^{2-4}, E$. coli continúa siendo una causa importante y quizá la más frecuente de infecciones amenazantes en nuestro medio ${ }^{5,6}$.

En población adulta podría considerarse el tracto urinario como el principal foco de infección por este microorganismo; otras fuentes de bacteriemia que conducen a cuadros sépticos pueden mostrar diferencias importantes en términos clínicos y microbiológicos. Un estudio checo publicado el año $2017^{7}$, en el que se hizo un perfil genético de 314 aislados de E. coli en hemocultivo para determinar los factores de virulencia comparados con aislados de E. coli en materia fecal, encontró mayor frecuencia de factores de virulencia en las cepas de hemocultivo, entre las que se especifican adhesinas y genes que codifican para captación de hierro. Adicionalmente, se encontró que los hemocultivos de pacientes con foco primario en el tracto genito-urinario contenían las cepas con mayor virulencia, comparados con hemocultivos de pacientes con leucemia, enfermedades del tracto digestivo u otros focos de infección. Dado que las implicaciones clínicas de estos hallazgos no son claras, el objetivo del presente estudio es determinar las características clínicas y microbiológicas de los pacientes con sepsis grave y choque séptico por $E$. coli, con o sin bacteriemia, con especial énfasis en las infecciones del tracto urinario que conducen a bacteriemia y sus diferencias potenciales con infecciones originadas en focos no urinarios.
'Universidad de Antioquia. Medellín, Colombia.

22Dirección de Investigaciones, Hospital Universitario San Vicente Fundación. Medellín, Colombia. Establecimientos de investigación: Hospital Universitario San Vicente Fundación (HUSVF), Hospital Pablo Tobón Uribe (HPTU) e IPS Universitaria León XIII (IPSU).

Conflictos de interés: ninguno que declarar.

Financiación: Universidad de Antioquia y el Departamento Administrativo de Ciencia, Tecnología e Innovación de Colombia (COLCIENCIAS código 111-5569-33362)

Recibido: 27 de febrero de 2018 Recibido (segunda versión): 15 de abril de 2019

Aceptado: 2 de julio de 2019

Correspondencia a:

Camilo Restrepo Álvarez camilo.75@hotmail.com 


\section{Métodos y participantes}

\section{Diseño y financiación}

Análisis secundario de datos obtenidos de un estudio de cohorte, prospectivo, multicéntrico, que se llevó a cabo entre julio 1 de 2014 y febrero 29 de 2017, cuyo objetivo era determinar el efecto de cada una de las estrategias del protocolo de reanimación con metas tempranas en la mortalidad intrahospitalaria en pacientes con sepsis grave y choque séptico. Dicho protocolo fue aprobado y financiado por la Universidad de Antioquia y el Departamento Administrativo de Ciencia, Tecnología e Innovación de Colombia (COLCIENCIAS, código 111-5569-33362; contrato 580-2013).

\section{Marco}

Servicio de urgencias y unidades de cuidado crítico de tres hospitales universitarios de alto nivel de complejidad de Medellín (Colombia): Hospital Universitario San Vicente Fundación (HUSVF), Hospital Pablo Tobón Uribe (HPTU) e IPS Universitaria León XIII (IPSU). El estudio fue aprobado por los comités de ética de las tres instituciones y se solicitó consentimiento informado a todos los participantes.

\section{Criterios de inclusión}

Pacientes mayores de 18 años, admitidos por el servicio de urgencias con diagnóstico registrado de sepsis grave o choque séptico, según consenso vigente para ese entonces del $2001^{8}$ y crecimiento en hemocultivos o urocultivos de $E$. coli, incluyendo los cultivos polimicrobianos en los que este microorganismo estuviese implicado. Se definió sepsis grave, de acuerdo a dichas definiciones", como sospecha o confirmación de infección con al menos dos criterios del síndrome de respuesta inflamatoria sistémica y por lo menos uno de los siguientes criterios de disfunción orgánica: Glasgow $<15 ; \mathrm{PaO}_{2} / \mathrm{FiO}_{2}<300$ o necesidad de ventilación mecánica; gasto urinario $<0,5$ $\mathrm{ml} / \mathrm{kg} / \mathrm{h}$ por dos horas reportado en la historia clínica; creatinina sérica $>2 \mathrm{mg} / \mathrm{dL}$ sin antecedente de enfermedad renal previa o aumento de $0,5 \mathrm{mg} / \mathrm{dL}$ con respecto a valores previos; INR $>1,5$ o TPT $>60$ segundos; íleo (descrito en la historia clínica); plaquetas $<150.000$ céls/ $\mathrm{mm}^{3}$; bilirrubinemia total $>2 \mathrm{mg} / \mathrm{dL}$; hiperlactatemia $>2$ $\mathrm{mmol} / \mathrm{L}$; llenado capilar lento o mayor de dos segundos; presión arterial sistólica (PAS) $<90 \mathrm{~mm} \mathrm{Hg}$ o presión arterial media $(\mathrm{PAM})<70 \mathrm{~mm} \mathrm{Hg}$ durante las primeras seis horas desde el ingreso. Se definió choque séptico como PAS persistentemente $<90 \mathrm{~mm} \mathrm{Hg}$ luego de administrado un bolo de cristaloides de mínimo $20 \mathrm{ml} / \mathrm{kg}$, o una concentración de lactato sérico $>4 \mathrm{mmol} / \mathrm{L}$.

\section{Criterios de exclusión}

Negativa del paciente, su familia o el médico tratante para participar en el estudio; diagnósticos concurrentes de embarazo, infarto del miocardio, asma aguda, arritmia de cualquier tipo, trauma, hemorragia gastrointestinal, convulsiones no asociadas a meningitis, sobredosis de sustancias psicoactivas, necesidad de cirugía en las primeras $24 \mathrm{~h}$, quemaduras, recuento de CD4 $<50$ céls $/ \mathrm{mm}^{3}$, estado hiperosmolar o cetoacidosis diabética, cirrosis; alta o remisión del hospital en las primeras $24 \mathrm{~h}$ de internación; participación previa en el estudio; paciente remitido de otra institución donde hubiese estado hospitalizado por más de 24 h; o pacientes con orden de no reanimación.

\section{Variables de interés}

Características clínicas. Con base en la historia clínica de ingreso, se recolectó información de las características demográficas, co-morbilidades, signos vitales, diagnóstico de ingreso, foco infeccioso de acuerdo a los criterios del CDC de Atlanta, E.U.A. ${ }^{10}$, sitio de aislamiento bacteriano, antimicrobianos administrados en las primeras $24 \mathrm{~h}$, obtención de hemocultivos previo a la primera dosis de antimicrobiano; además, los paraclínicos de las primeras seis horas de atención para el cálculo de los puntajes SOFA y APACHE II. Los resultados de laboratorio faltantes o no solicitados para los cálculos de estos puntajes se asumieron como normales?.

Características microbiológicas. Se diferenciaron tres grupos: aislamiento de E. coli en hemocultivos, E. coli en urocultivo y $E$. coli en ambas muestras. Se incluyeron también los cultivos reportados como polimicrobianos que incluía $E$. coli dentro de los microorganismos aislados. Se consignaron los antimicrobianos reportados por el antibiograma y el perfil de sensibilidad (sensible, indeterminado o resistente).

\section{Desenlaces}

En el análisis clínico de los tres grupos descritos se evaluó la necesidad de Unidad de Cuidados Intensivos (UCI)/Unidad de Cuidados Especiales (UCE) (ICU: Intensive Care Unity PCU: Progressive Care Unit, por sus siglas en inglés) o ventilación mecánica, decisión que fue tomada por el equipo médico a cargo del paciente, sin ninguna influencia o participación de los investigadores. El estado vital del paciente (vivo o muerto), se evaluó en el hospital de forma rutinaria durante todo el tiempo de estancia y al momento del alta. Dentro del análisis microbiológico se consideró como desenlace principal la presencia de bacterias productoras de $\beta$-lactamasas de espectro extendido (BLEE), dependiendo del foco infeccioso y el tratamiento antimicrobiano empírico

\section{Fuente de los datos}

Auxiliares de investigación entrenados en cada institución realizaron todo el proceso de cribado, selección de los pacientes y recolección de la información por medio 
de un formulario estandarizado y de manera prospectiva. Los co-investigadores revisaron los datos recogidos y los pacientes incluidos por los auxiliares de investigación. Para la identificación de los pacientes se cribó a todos los que ingresaron por urgencias con diagnóstico de infección, sepsis, sepsis grave o choque. Las definiciones: fuente de la infección, disfunción orgánica o de choque, se verificaron con base en los datos extraídos de los registros clínicos de las primeras seis horas, al igual que los datos referentes al proceso de diagnóstico y tratamiento, incluyendo tiempo.

\section{Análisis estadístico}

Se realizó un análisis descriptivo de las variables demográficas, clínicas y microbiológicas. Las variables continuas se describieron usando medianas y rangos intercuartílicos, las variables categóricas por medio de proporciones. La comparación entre grupos para las variables continuas se realizó por medio de la prueba Kruskal Wallis y para las variables categóricas con la prueba de $\chi^{2}$. Con respecto a la población de estudio, se compararon las variables de los pacientes según su distribución en los siguientes grupos:

- Con aislamiento de E. coli sólo en hemocultivo.

- Con aislamiento sólo en urocultivo.

- Con aislamiento en ambas muestras.

Así mismo, dentro de cada uno de los anteriores grupos se comparó la mortalidad con base en los antimicrobianos empíricos más comúnmente prescritos. Se consideró significativo un valor de $\mathrm{p}<0,05$ y no se hicieron ajustes por múltiples pruebas.

\section{Resultados}

Participantes. En la cohorte inicial de estudio se reclutaron 2.510 pacientes y se le solicitó hemocultivos a $87,1 \%(n=2.185)$ de ellos, de los cuales $29,1 \%(n=635)$ fueron positivos y en $37,2 \%(\mathrm{n}=236)$ se aisló $E$. coli. Se solicitó urocultivo a 45,8\% $(\mathrm{n}=1.148)$ de los casos, de los cuales $51 \%(\mathrm{n}=585)$ fueron positivos y $57,3 \%$ $(\mathrm{n}=335)$ fueron positivos para E. coli. De los anteriores, 113 pacientes tuvieron tanto hemocultivos como urocultivo positivos, para un total de población de estudio de 458 pacientes: 123 con aislamiento de $E$. coli sólo en el hemocultivo, 222 con crecimiento sólo en el urocultivo y 113 en ambas muestras. La mayor proporción fueron mujeres $(n=293 ; 64 \%)$, y la mediana de la edad fue de 64 años $($ RIQ $=48-76)($ Tabla 1$)$.

\section{Principales características}

Durante la valoración inicial en el servicio de urgencias, la mayoría de los pacientes $(\mathrm{n}=339 ; 74 \%)$ fueron ingresados con un diagnóstico inicial de infección urinaria. Sin embargo, en el grupo de pacientes con sólo hemocultivos positivos, el sitio primario de infección fue principalmente el torrente sanguíneo $(n=47 ; 38,2 \%)$ seguido de infección intra-abdominal $(n=38 ; 30,9 \%)$. La neumonía, la infección de piel y tejidos blandos, otras infecciones, las infecciones urinarias y las de fuente desconocida representaron el restante $30,9 \%(n=38)$ de este grupo. Con la excepción del aumento en la creatinina sérica y la alteración del estado mental detectada por medio de la escala de Glasgow, los pacientes con aislamiento de $E$. coli sólo en hemocultivo tuvieron un mayor porcentaje de parámetros de disfunción de órganos que los pacientes con sólo urocultivo o con hemocultivo y urocultivo positivos. Esta diferencia fue particularmente notable en la frecuencia de hiperbilirrubinemia y trombocitopenia (Tabla 1).

Las co-morbilidades más frecuentes fueron diabetes mellitus en 129 pacientes $(28,2 \%), 71(15,5 \%)$ de estos con complicaciones crónicas, y enfermedad renal en 70 pacientes $(15,3 \%)$. Los pacientes con hemiplejía o paraplejía presentaban con mayor frecuencia aislamiento de $E$. coli sólo en el urocultivo $(\mathrm{n}=24 ; 10,8 \%)$, mientras los pacientes con cualquier neoplasia, incluyendo leucemia y linfoma, tenían mayor frecuencia de aislamiento de $E$. coli en el hemocultivo $(\mathrm{n}=28 ; 22,8 \%)$.

Los pacientes con aislamiento de $E$. coli únicamente en hemocultivo tuvieron puntajes SOFA y APACHE II más altos [5 (RIQ=3-8) y $15(\mathrm{RIQ}=11-19)$, respectivamente], y peor pronóstico dado por mayor frecuencia de ingreso a UCI $(\mathrm{n}=63 ; 51 \%)$, mayor necesidad de ventilación mecánica $(\mathrm{n}=19 ; 15,5 \%)$, mayor mortalidad y estancia hospitalaria $(\mathrm{n}=22 ; 18 \%$; mediana de 12 días, $\mathrm{RIQ}=7-17$, respectivamente) (Tabla 1$)$.

La proporción de pacientes tratados empíricamente con carbapenémicos vs no carbapenémicos, sin embargo, fue similar en los tres grupos (hemocultivos 12,8 vs $87,2 \%$, urocultivos 11,4 vs $88,6 \%$ y en hemocultivo y urocultivos 5,7 vs 94,3\%). Estas frecuencias de prescripción de antimicrobianos empíricos, por otra parte, no estuvieron asociadas con diferencias estadísticamente significativas en la mortalidad (Tabla 2). Adicionalmente, la frecuencia de bacterias productoras de BLEE fue de $17 \%(n=77)$, siendo menor en los pacientes con sólo hemocultivo positivo ( $\mathrm{n}=11 ; 8,9 \%)$, en comparación con los urocultivos o ambas muestras $(\mathrm{n}=46 ; 20,7 \%$ y $n=20 ; 17,7 \%$, respectivamente).

En los hallazgos clínicos y de laboratorio al ingreso, se apreció una tendencia a mayor temperatura, leucocitosis y valores de creatinina sérica en pacientes con aislamiento del microorganismo en sangre y orina, mientras el compromiso hemodinámico, los valores de lactato y la oxigenación tuvieron un peor comportamiento en los pacientes con bacteriemia aislada. 


\begin{tabular}{|c|c|c|c|c|}
\hline Variable & $\begin{array}{l}\text { E. coli en hemocultivo } \\
\text { n: } 123(26,8 \%)\end{array}$ & $\begin{array}{l}\text { E. coli en urocultivo } \\
\text { n: } 222(48,5 \%)\end{array}$ & $\begin{array}{l}\text { E. coli en ambos } \\
\text { n: } 113(24,7 \%)\end{array}$ & Valor de $\mathrm{p}$ \\
\hline Género femenino & $79(64,2 \%)$ & $146(65,8 \%)$ & $68(60,2 \%)$ & 0,601 \\
\hline Edad* & $62(48-76)$ & $65(44-76)$ & $65(51-77)$ & 0,6485 \\
\hline \multicolumn{5}{|l|}{ Infección principal } \\
\hline ITU & $4(3,25 \%)^{* *}$ & $222(100 \%)$ & $113(100 \%)$ & 0,001 \\
\hline \multicolumn{5}{|l|}{ Criterios de disfunción de órganos } \\
\hline Lactato $>2(n=425)$ & $88(75,2 \%)$ & $124(62 \%)$ & $71(65,7 \%)$ & 0,054 \\
\hline $\mathrm{PaO}_{2} / \mathrm{FiO}_{2}<300(\mathrm{n}=367)$ & $57(52,3 \%)$ & $59(35,5 \%)$ & $37(40,2 \%)$ & 0,021 \\
\hline Aumento de creatinina $>0,5$ o creatinina $>2 \mathrm{mg} / \mathrm{dl}(\mathrm{n}=455)$ & $44(36,1 \%)$ & $51(23,2 \%)$ & $45(39,8 \%)$ & 0,003 \\
\hline Bilirrubina $>2(n=256)$ & $44(48,9 \%)$ & $7(6,7 \%)$ & $8(12,9 \%)$ & 0,001 \\
\hline $\mathrm{PAM}<70$ & $60(48,8 \%)$ & $90(40,5 \%)$ & $42(37,2 \%)$ & 0,165 \\
\hline Plaquetas $/ \mathrm{mm}^{3}<150.000(\mathrm{n}=455)$ & $48(39 \%)$ & $30(13,7 \%)$ & $29(25,7 \%)$ & 0,001 \\
\hline Glasgow $<15$ & $14(11,4 \%)$ & $22(9,9 \%)$ & $16(14,2 \%)$ & 0,511 \\
\hline INR $>1,5(n=392)$ & $22(19 \%)$ & $21(11,5 \%)$ & $9(9,7 \%)$ & 0,090 \\
\hline TPT $>60(n=391)$ & $3(2,6 \%)$ & $2(1,1 \%)$ & $1(1,1 \%)$ & 0,537 \\
\hline \multicolumn{5}{|l|}{ Co-morbilidades } \\
\hline Insuficiencia cardíaca congestiva & $7(5,7 \%)$ & $14(6,3 \%)$ & $9(8 \%)$ & 0,764 \\
\hline Demencia & $2(1,6 \%)$ & $6(2,7 \%)$ & $4(3,5 \%)$ & 0,651 \\
\hline Hemiplejía o paraplejia & $4(3,3 \%)$ & $24(10,8 \%)$ & $4(3,5 \%)$ & 0,008 \\
\hline Enfermedad renal & $17(13,8 \%)$ & $37(16,7 \%)$ & $16(14,2 \%)$ & 0,726 \\
\hline Cualquier neoplasia, incluyendo leucemia y linfoma & $28(22,8 \%)$ & $24(10,8 \%)$ & $11(9,7 \%)$ & 0,003 \\
\hline Enfermedad pulmonar crónica & $13(10,6 \%)$ & $30(13,5 \%)$ & $15(13,3 \%)$ & 0,715 \\
\hline Diabetes mellitus con complicaciones crónicas & $19(15,5 \%)$ & $36(16,2 \%)$ & $16(14,2 \%)$ & 0,886 \\
\hline Diabetes mellitus sin complicaciones & $13(10,6 \%)$ & $24(10,8 \%)$ & $21(18,6 \%)$ & 0,093 \\
\hline \multicolumn{5}{|l|}{ Gravedad } \\
\hline Índice Charlson* & $1(0-2)$ & $1(0-2)$ & $1(0-2)$ & 0,4854 \\
\hline Total Puntaje SOFA* & $5(3-8)$ & $3(1-4)$ & $4(2-5)$ & 0,0001 \\
\hline Total APACHE II* & $15(11-19)$ & $11(7-16)$ & $14(10-17)$ & 0,0001 \\
\hline \multicolumn{5}{|l|}{ Desenlaces } \\
\hline Ingreso a UCI/UCE & $63(51,2 \%)$ & $56(25,2 \%)$ & $48(42,5 \%)$ & 0,001 \\
\hline Ventilación mecánica & $19(15,5 \%)$ & $10(4,5 \%)$ & $7(6,2 \%)$ & 0,001 \\
\hline Mortalidad hospitalaria & $22(18 \%)$ & $8(3,6 \%)$ & $7(6,2 \%)$ & 0,001 \\
\hline Estancia hospitalaria* & $12(7-17)$ & $7(5-12)$ & $10(7-14)$ & 0,0001 \\
\hline
\end{tabular}

Un total de 424 pacientes $(92,6 \%)$ recibieron manejo antimicrobiano en las primeras $24 \mathrm{~h}$, más frecuentemente con piperacilina/tazobactam $(n=256 ; 60,3 \%)$ y en segundo lugar con aztreonam $(n=76 ; 17,9 \%)$. Un comportamiento similar se observó en los pacientes con aislamiento de $E$. coli sólo en urocultivo ( $\mathrm{n}=111 ; 55,2 \%$ y n $=52 ; 25,9 \%$, respectivamente) y en ambas muestras ( $\mathrm{n}=68 ; 64,2 \%$ y $\mathrm{n}=20 ; 18,9 \%$, respectivamente). En los pacientes con sólo hemocultivo positivo para E. coli, en cambio, el segundo antimicrobiano fue meropenem $(\mathrm{n}=15 ; 12,8 \%)$ (Tabla 3$)$.

\section{Discusión}

Nuestros resultados sugieren que la infección por $E$. coli más frecuentemente asociada a sepsis es el foco urinario, como se ha evidenciado en otros estudios ${ }^{1}$. Sin embargo, E. coli también puede estar asociada a otros focos de infección, con neumonía y las infecciones intraabdominales como frecuentes focos en estos pacientes ${ }^{11}$.

El alto número de infecciones del tracto urinario (ITUs) podría estar relacionado con la presencia de algunas co-morbilidades que predisponen en gran 


\begin{tabular}{|c|c|c|c|c|}
\hline $\begin{array}{l}\text { Mortalidad hospitalaria } \\
\text { (número fallecidos/población del subconjunto) }\end{array}$ & $\begin{array}{l}\text { E. coli en hemocultivo } \\
\text { n: } 123(26,8 \%)\end{array}$ & $\begin{array}{l}\text { E. coli en urocultivo } \\
\text { n: } 222(48,5 \%)\end{array}$ & $\begin{array}{l}\text { E. coli en ambos } \\
\text { n: } 113(24,7 \%)\end{array}$ & Valor de $p^{*}$ \\
\hline Carbapenémicos $(n=44 ; 10,3 \%)$ & $0 / 15(0 \%)$ & $0 / 23(0 \%)$ & $0 / 6(0 \%)$ & \multirow{3}{*}{0,189} \\
\hline Piperacilina/tazobactam ( $n=256,60,3 \%)$ & $18 / 77(23,4 \%)$ & $4 / 111(3,6 \%)$ & $4 / 68(5,9 \%)$ & \\
\hline Otros $(n=124 ; 29,2 \%)$ & $4 / 31(12,9 \%)$ & $4 / 88(4,6 \%)$ & $3 / 39(7,7 \%)$ & \\
\hline
\end{tabular}

*Para la comparación de proporciones de mortalidad hospitalaria según cultivo y antimicrobianos empíricos. **Para la comparación de proporciones de mortalidad hospitalaria según antimicrobiano empírico, dentro de cada grupo de cultivos.

\begin{tabular}{|c|c|c|c|c|}
\hline Variable & $\begin{array}{l}\text { E. coli en hemocultivo } \\
\text { n: } 123(26,8 \%)\end{array}$ & $\begin{array}{l}\text { E. coli en urocultivo } \\
\text { n: } 222(48,5 \%)\end{array}$ & $\begin{array}{l}\text { E. coli en ambos } \\
\text { n: } 113(24,7 \%)\end{array}$ & Valor de $p$ \\
\hline PAS $(\mathrm{mm} / \mathrm{Hg})$ & $100(81-120)$ & $107(85-125)$ & $110(88-137)$ & 0,0632 \\
\hline Temperatura $\left({ }^{\circ} \mathrm{C}\right)$ & $37,3(36,9-38,5)$ & $37,5(36,8-38,7)$ & $38(37-39)$ & 0,0959 \\
\hline Frecuencia cardiaca (lat/min) & $110(97-126)$ & $104(88-120)$ & $108(90-123)$ & 0,0233 \\
\hline Frecuencia respiratoria (resp/min) & $20(18-24)$ & $18(17-20)$ & $19(18-36)$ & 0,0001 \\
\hline Leucocitos $/ \mathrm{mm}^{3}$ & $13.520(7.420-19.600)$ & $13.350(9.300-17.100)$ & $14.500(9.800-20.100)$ & 0,1641 \\
\hline Lactato (mmol/L) & $3,1(2,1-4,2)$ & $2.4(1,5-3,2)$ & $2.6(1,8-3,5)$ & 0,0001 \\
\hline Creatinina (mg/dL) & $1,3(0,9-2,3)$ & $1(0,8-1,7)$ & $1.5(1,1-2,1)$ & 0,0001 \\
\hline Bilirrubina (mg/dL) & $2.1(0,9-4,2)$ & $0.7(0,5-1)$ & $1.1(0,7-1,5)$ & 0,0001 \\
\hline Índice $\mathrm{PaO}_{2} / \mathrm{FiO}_{2}$ & $292(238-363)$ & $327(262-390)$ & $320(260-360)$ & 0,1877 \\
\hline Antimicrobianos en las primeras $24 \mathrm{~h}$ de ingreso & $117(95,1 \%)$ & $201(90,5 \%)$ & $106(93,8 \%)$ & 0,253 \\
\hline Piperacilina/tazobactam & $77(65,8 \%)$ & $111(55,2 \%)$ & $68(64,2 \%)$ & \\
\hline Aztreonam & $4 \quad(3,4 \%)$ & $52(25,9 \%)$ & $20(18,9 \%)$ & \\
\hline Meropenem & $15(12,8 \%)$ & $22(11 \%)$ & $6(5,7 \%)$ & \\
\hline Ciprofloxacina & $4(3,4 \%)$ & $4(2 \%)$ & $4 \quad(3,8 \%)$ & \\
\hline Vancomicina & $5(4,3 \%)$ & $3(1,5 \%)$ & $2(1,9 \%)$ & \\
\hline Ampicilina/sulbactam & $2(1,7 \%)$ & $5(2,5 \%)$ & $2(1,9 \%)$ & \\
\hline Claritromicina & $3(2,6 \%)$ & $2(1 \%)$ & - & \\
\hline Ceftriaxona & $2 \quad(1,7 \%)$ & $1 \quad(0,5)$ & $1 \quad(0,9 \%)$ & \\
\hline Cefepime & $1 \quad(0,9 \%)$ & - & $2 \quad(1,9 \%)$ & \\
\hline Otros* & $4(0,9 \%)$ & $1(0,5 \%)$ & - & \\
\hline
\end{tabular}

medida a este tipo de infecciones, como son la diabetes mellitus $^{12}$ y la enfermedad renal crónica ${ }^{13}$. El enfoque inicial de la mayor parte de los pacientes de nuestro estudio, independientemente del foco inicial, incluyó la solicitud de hemocultivos y el inicio de manejo antimicrobiano de amplio espectro dentro de la primera hora, como se recomienda según las guías Surviving Sepsis
Campaign (SSC) ${ }^{14}$. A pesar de algunas recomendaciones para iniciar tratamiento empírico con aminopenicilinas, fluoroquinolonas de segunda generación, cefalosporinas de tercera generación y carbapenémicos ${ }^{12}$, la mayor parte de nuestros pacientes recibió manejo inicial con piperacilina/tazobactam. Esto podría estar relacionado con el perfil de resistencia institucional; para el año 
2016 sólo $35,3 \%$ de las cepas de $E$. coli eran sensibles a ampicilina y $44,2 \%$ a ampicilina/sulbactam en pacientes que no estaban en UCI; con valores de 30,7 y 41,7\%, respectivamente, para pacientes en UCI (15). Estudios recientes en otros países han demostrado un aumento de las cepas no susceptibles a piperacilina/tazobactam, desde $6,8 \%$ para el año 2005 a $23,8 \%$ en el $2014^{16}$. Sin embargo, en reportes locales, la susceptibilidad de $E$. coli a piperacilina/tazobactam se ha mantenido estable durante los últimos tres años, con una susceptibilidad de 92, 91,9 y 92,1\% para los años 2014, 2015 y 2016, respectivamente ${ }^{17}$, lo que es similar al porcentaje de resistencia encontrado en nuestro estudio. Este perfil de resistencia de E. coli, sumado a la gravedad de los pacientes incluidos en este estudio, justificaría el uso empírico de esta clase de antimicrobianos.

En cuanto a los carbapenémicos, tratamiento que se reserva para pacientes con E. coli productora de BLEEs ${ }^{18}$, se encontró un porcentaje muy bajo de resistencia a esta clase de antimicrobianos, lo que los convierte en una buena herramienta para el tratamiento empírico en pacientes con sepsis o choque séptico. Sin embargo, en un estudio de cohorte retrospectivo, efectuado entre 2011 y 2013, en dos hospitales universitarios de Singapur, con 394 pacientes y diagnóstico de bacteriemia por E. coli y Klebsiella pneumoniae productores de BLEEs de foco biliar y urinario, que buscaba evaluar la mortalidad a 30 días entre quienes recibían tratamiento empírico con piperacilina/tazobactam $(n=94)$ o carbapenémicos $(n=57)$, la diferencia entre la mortalidad de estos dos grupos no fue significativa, siendo de $40(70,2 \%)$ y de $49(52,1 \%)$ pacientes, respectivamente, con un valor de $p=0,89$. En el mismo estudio, luego del análisis multivariable, se encontró que el tratamiento empírico con piperacilina/tazobactam no aumentaba la mortalidad a 30 días (OR: 1,00, IC 95\%: 0,45-2,17) y, adicionalmente, este tratamiento estaba a asociado a una menor adquisición de infecciones por hongos o bacterias multi-droga-resistentes $(7$ [7,4\%] vs $14[24,6 \%]), p<0,01)$ mientras que el tratamiento con carbapenémicos se asoció a mayor riesgo de contraer este tipo de infecciones (OR 3,32, IC 95\%: 1,12-9,87). Lo anterior apoya el uso de piperacilina/tazobactam por encima de carbapenémicos como primera línea de manejo para el tratamiento empírico en pacientes con sepsis o choque séptico. Por otro lado, un pequeño estudio $(\mathrm{n}=79)$ en pacientes con bacteriemia de origen urinario por $E$. coli y $K$. pneumoniae realizado en la región de Calgary (Canadá), sugirió que el tratamiento empírico con betalactámicos + inhibidor de betalactamasas estaría asociado con mayor mortalidad, comparado con aquellos que recibieron tratamiento un antimicrobiano diferente ${ }^{19}$. Un estudio similar al canadiense, con igual número de pacientes, pero con bacteriemia por E. coli de foco no urinario, asoció piperacilina/tazobactam con mayor mortalidad a 90 días cuando se le comparó con carbapenémicos $(\mathrm{OR}=7,9$; IC 95\%: 1,2-53) ${ }^{20}$.

En nuestro estudio no se encontró un porcentaje muy alto de E. coli productoras de BLEE en los hemocultivos, comparado con otros estudios ${ }^{1}$. Sin embargo, encontramos una mayor proporción de cepas con BLEEs en los aislados provenientes de urocultivos o ambas muestras; lo que se correlaciona adecuadamente con los datos locales, donde $17,8 \%$ de los aislados de $E$. coli en UCI y $14,2 \%$ no UCI, producen $\mathrm{BLEEs}^{21}$. Por tanto, para efectos del inicio empírico de antimicrobiano al sospechar una infección por cepas productores de BLEE, parecería razonable tener en cuenta en la decisión la sospecha de foco urinario, adicional a otros factores de riesgo como son la edad mayor de 60 años, un índice de Charlson mayor a 2, tratamiento antimicrobiano previo, el cateterismo urinario y la infección asociada a los cuidados de la salud ${ }^{18}$.

Por lo anterior, se considera el foco infeccioso en los pacientes con sepsis grave y choque séptico un aspecto fundamental para dirigir el tratamiento empírico; en aquellos pacientes en quien se sospeche foco urinario, como foco primario, se tiene como preferencia actualmente el uso de piperacilina/tazobactam o carbapenémicos ${ }^{17,18,20}$, como vimos en nuestro estudio, evaluando también los factores de riesgo para cepas que producen BLEEs y la susceptibilidad local para escoger entre uno u otro. Queda la hipótesis, de si otros antimicrobianos de menor espectro pero, de amplio uso, como es aztreonam, podrían tener igual o mejores desenlaces a los antimicrobianos usualmente ordenados, teniendo en cuenta solamente el foco infeccioso, en este caso las infecciones del tracto urinario. Es importante resaltar que la selección empírica del antimicrobiano es sólo el primer paso para asegurar un adecuado tratamiento, dado que éste debe ser individualizado para cada paciente y ajustado periódicamente; debido, entre otras razones, a variables de farmacocinética y farmacodinamia que podrían afectar su efectividad ${ }^{22,23}$.

Aunque estudio previos han demostrado que la infección por bacterias productoras de BLEE está asociada a una alta mortalidad ${ }^{24}$, en nuestro estudio este desenlace fue mayor en los pacientes con aislamiento de E. coli sólo en hemocultivos, grupo que también presentaba el menor porcentaje de cepas con BLEEs como se mencionó previamente. Es necesario hacer un análisis multivariable para poder determinar si la presencia de BLEEs está asociada o no con mayor o menor mortalidad, en un estudio diseñado para tal fin.

Por último, es importante resaltar que los pacientes con sólo hemocultivos positivos presentaron con más frecuencia hiperlactatemia, hiperbilirrubinemia, trombocitopenia e hipotensión arterial no respondedora a líquidos; todo lo cual podría estar asociados a la alta mortalidad en este grupo de pacientes. Es importante, por tanto, en estudios posteriores indagar sobre predictores de mortalidad y 
otras variables para definir presencia y gravedad de hipoperfusión en pacientes con sepsis o choque séptico ${ }^{25-28}$.

Una de las principales limitaciones del estudio está relacionada con el carácter retrospectivo de la información, debido a que en la cohorte original no se planteaba específicamente analizar el pronóstico según el tipo y la fuente de infección o su microbiología. Por otra parte, la fuente de infección en los pacientes con bacteriemia aislada se analizó de acuerdo con el proceso diagnóstico del criterio de los médicos tratantes y no por medio de un protocolo estandarizado para la investigación. Por lo anterior, no se puede descartar algo de clasificación inadecuada con respecto a la verdadera fuente de infección en este subgrupo. Otra limitación es el análisis local de los datos, debido a que algunos hallazgos pueden no ser extrapolables a otra población por corresponder a una característica epidemiológica local, como la mayor presencia de cepas productoras de BLEE en infecciones de foco urinario.

\section{Conclusiones}

Escherichia coli continúa siendo una causa importante de sepsis en nuestro medio, con la infección urinaria como el principal diagnóstico asociado a este microorganismo. Según los hallazgos de este estudio, los pacientes con aislamiento de $E$. coli sólo en hemocultivo presentan con mayor frecuencia desenlaces desfavorables, como mayor admisión a UCI, estancia hospitalaria y mortalidad, en comparación con los pacientes con E. coli en urocultivo con o sin bacteriemia. Adicionalmente, en este grupo de pacientes se observó una menor frecuencia de cepas que producen BLEE por lo que se propone utilizar el foco infeccioso, sumado a factores de riesgo para cepas con BLEE, para dirigir el tratamiento antimicrobiano. Estudios posteriores en nuestra población deben explorar el papel de las cepas productoras de BLEE en el pronóstico de este tipo de pacientes.

Agradecimientos. A los pacientes y sus familias.

\section{Resumen}

Introducción: Escherichia coli es causa frecuente de un amplio espectro de infecciones, desde una infección urinaria no complicada hasta la sepsis grave y el choque séptico, asociadas con desenlaces de alto impacto como ingreso a UCI y mortalidad. Objetivos: Determinar las diferencias en mortalidad, ingreso a UCI/UCE, presencia de cepas BLEE y tratamiento antimicrobiano en pacientes con sepsis grave y choque séptico por $E$. coli, con o sin bacteriemia, así como su variabilidad dependiendo del foco infeccioso. Material y Métodos: Análisis secundario de estudio de cohorte prospectivo multicéntrico. Resultados: De 458 pacientes que tenían infección por E. coli, 123 tenían aislamiento sólo en hemocultivo, 222 sólo en urocultivo y 113 en ambas muestras. El aislamiento sólo en hemocultivo se asoció mayor frecuencia de ingreso a UCI $(n=63 ; 5,2 \%)$, mayor necesidad de ventilación mecánica $(\mathrm{n}=19 ; 15,5 \%)$, mayor mortalidad y estancia hospitalaria $(n=22 ; 18 \%$; mediana de 12 días, $\mathrm{RIQ}=7-17$, respectivamente), pero con menor presencia de cepas productoras de BLEE en comparación con urocultivos y hemocultivo/urocultivo ( $n=20 ; 17,7 \%$ y $n=46 ; 20,7 \%$, respectivamente). Recibieron tratamiento antimicrobiano en las primeras $24 \mathrm{~h}$ 424 pacientes $(92,6 \%)$, con más frecuencia piperacilina/ tazobactam $(n=256,60,3 \%)$. La proporción de pacientes tratados empíricamente con carbapenémicos vs no carbapenémicos fue similar en los tres grupos. Discusión: El foco infeccioso, sumado a factores de riesgo para cepas productoras de BLEE, son herramientas útiles para definir pronóstico y tratamiento en esta población, debido a la variabilidad clínica y microbiológica en los distintos aislados. Conclusión: Los pacientes con aislamiento de E. coli sólo en hemocultivo presentan con mayor frecuencia desenlaces desfavorables en comparación con los pacientes con $E$. coli en urocultivo, con o sin bacteriemia. Llama la atención en nuestro medio la menor cantidad de cepas productoras de BLEE en los pacientes con sólo hemocultivo positivo.

\section{Referencias bibliográficas}

1.- Peralta G, Lamelo M, Alvarez-García P, Velasco M, Delgado A, Horcajada J P, et al. Impact of empirical treatment in extendedspectrum beta-lactamase-producing Escherichia coli and Klebsiella spp. bacteremia. A multicentric cohort study. BMC Infect Dis. 2012;12:245. doi: 10.1186/1471-2334-12-245.

2.- Donnenberg M S. Capítulo 220: Enterobacteriaceae. En: Mandell GL, Bennett JE, Dolin R, Blase MJ, editors. Mandell, Douglas y Bennett. Enfermedades Infecciosas.
Principios y Práctica. 8va Edición. España. Elsevier 2016, p. 2640-55.

3.- Diekema D J, Beekmann S E, Chapin K C, Morel K A, Munson E, Doern G V. Epidemiology and outcome of nosocomial and community-onset bloodstream infection. J Clin Microbiol 2003; 41 (8): 3655-60. doi: 10.1128/ jcm.41.8.3655-3660.2003.

4.- Artico M J, Rocchi M, Gasparotto A, Ocaña Carrizo V, Navarro M, Mollo V, et al. Bacteriemias de origen comunitario en pacientes adultos que acuden al servicio de urgencias de un hospital universitario. Rev
Argentina Microbiol 2012; 44 (1): 10-5. http:// www.scielo.org.ar/pdf/ram/v44n1/v44n1a03. pdf.

5.- Marra A R, Camargo L F, Pignatari A C, Sukiennik T, Behar P R, Medeiros E A, et al. Nosocomial bloodstream infections in Brazilian hospitals: analysis of 2,563 cases from a prospective nationwide surveillance study. J Clin Microbiol. 2011;49(5):1866-71. doi: 10.1128/JCM.00376-11.

6.- De La Rosa G, León A. L, Jaimes F. Epidemiología y pronóstico de pacientes con infección del torrente sanguíneo en 10 
hospitales de Colombia. Rev chil infectol 2016; 33 (2): 141-9. http://dx.doi.org/10.4067/S071610182016000200003

7.- Micenková L, Beňová A, Frankovičová L, Bosák J, Vrba M, Ševčíková A, et al. Human Escherichia coli isolates from hemocultures: Septicemia linked to urogenital tract infections is caused by isolates harboring more virulence genes than bacteraemia linked to other conditions. Int J Med Microbiol 2017; 307 (3): 182-9. doi: 10.1016/j.ijmm.2017.02.003.

8.- Levy M M, Fink M P, Marshall J C, Abraham E, Angus D, Cook D, et al. $2001 \mathrm{SCCM} /$ ESICM/ACCP/ATS/SIS International Sepsis Definitions Conference. Crit Care Med 2003; 31 (4): 1250-6. doi: 10.1097/01. CCM.0000050454.01978.3B

9.- Levy M M, Fink M P, Marshall J C, Abraham E, Angus D, Cook D, et al. International Sepsis Definitions Conference. 2001 SCCM/ ESICM/ACCP/ATS/SIS International Sepsis Definitions Conference. Intensive Care Med 2003; 29 (4): 530-8. doi:10.1097/01. CCM.0000050454.01978.3B.

10.- Horan T C, Andrus M, Dudeck M A. CDC/ NHSN surveillance definition of health careassociated infection and criteria for specific types of infections in the acute care setting. Am J Infect Control 2008; 36 (5): 309-32. https://www.hopkinsmedicine.org/armstrong_ institute/_files/clabsi_toolkit/Appendix_C_ CDC_NHSN_Definitions.pdf.

11.- Novosad S A, Sapiano M R, Grigg C, Lake J, Robyn M, Dumyati G, et al. Vital signs: epidemiology of sepsis: prevalence of health care factors and opportunities for prevention. MMWR Morb Mortal Wkly Rep 2016; 65: 864-9. doi: 10.15585/mmwr.mm6533e1.

12.- Dreger N M, Degener S, Ahmad-Nejad P, Wöbker G, Roth S. Urosepsis-etiology, diagnosis, and treatment. Dtsch Arztebl Int 2015; 112 (49): 837-47. doi: 10.3238/ arztebl.2015.0837.

13.- Sobotová D. Urinary tract infections and chronic renal failure]. Vnitr Lek. 2011; 57 (7-8): 626-30. doi: 10.3238/ arztebl.2015.0837.

14.- Dellinger R P, Levy M M, Rhodes A, Annane D, Gerlach H, Opal S M, et al. Surviving sepsis campaign: international guidelines for management of severe sepsis and septic shock: 2012. Crit Care Med 2013; 41 (2): 580-637. doi: 10.1097/CCM.0b013e31827e83af.

15.- Grupo Germen [Internet] Medellín, Colombia: Perfiles de sensibilidad a antibióticos de Escherichia coli en Servicios de UCI y NO UCI 2014-2015-2016 [Citado 02/03/2017]. Disponible en: http://www.grupogermen.org/ pdf/escherichia_coli_12_14.pdf.

16.- Stapleton P J, Lundon D J, McWade R, Scanlon N, Hannan M M, O’Kelly F, et al. Antibiotic resistance patterns of Escherichia coli urinary isolates and comparison with antibiotic consumption data over 10 years, 2005-2014. Ir J Med Sci 2017; 86 (3): 733-41. doi: 10.1007/s11845-016-1538-z.

17.- Grupo Germen [Internet] Medellín, Colombia: Perfiles de sensibilidad a antibióticos de Escherichia coli en Urgencias 2014-2015-2016 [Citado el 4 de marzo de 2017]. Disponible en: http://www.grupogermen.org/pdf/escherichia coli_12_14.pdf.

18.- García-Hernández A M, García-Vázquez E, Hernández-Torres A, Ruiz J, Yagüe G, Herrero $\mathrm{J}$ A, et al. Bacteraemia due to Escherichia coli producing extended-spectrum beta-lactamases (ESBL): clinical relevance and today's insights. Rev Esp Quimioter 2011; 24 (2): 57-66. PMID: 21666996.

19.- Chaubey V P, Pitout J D, Dalton B, Ross T, Church D L, Gregson D B, et al. Clinical outcome of empiric antimicrobial therapy of bacteremia due to extended-spectrum betalactamase producing Escherichia coli and Klebsiella pneumoniae. BMC Res Notes 2010; 3: 116. doi: 10.1186/1756-0500-3-116.

20.- Ofer-Friedman H, Shefler C, Sharma S, Tirosh A, Tal-Jasper R, Kandipalli D, et al. Carbapenems versus piperacillin-tazobactam for bloodstream infections of nonurinary source caused by extended-spectrum betalactamase-producing Enterobacteriaceae. Infect Control Hosp Epidemiol. 2015; 36(8): 981-5. doi: 10.1017/ice.2015.101.

21.- Grupo Germen [Internet] Medellín, Colombia: Porcentajes de aislamientos de Escherichia coli productores de betalactamasas de espectro extendido (BLEE) en los años 2014-2015-2016 según el tipo de localización [Citado el 4 de marzo de 2017]. Disponible en: http://www. grupogermen.org/pdf/escherichia_coli_12_14 pdf.

22.- Kollef M H. Antibiotics for the critically ill: more than just selecting appropriate initial therapy. Crit Care. 2013; 17(3): 146. doi: 10.1186/cc12698.

23.- Carlier M, Carrette S, Roberts JA, Stove V, Verstraete A, Hoste E, et al. Meropenem and piperacillin/tazobactam prescribing in critically ill patients: does augmented renal clearance affect pharmacokinetic/pharmacodynamic target attainment when extended infusions are used? Crit Care 2013; 17 (3): R84. doi: 10.1186/cc12705.

24.- Ray S, Anand D, Purwar S, Samanta A, Upadhye KV, Gupta P, et al. Association of high mortality with extended-spectrum $\beta$-lactamase (ESBL) positive cultures in community acquired infections. J Crit Care. 2017; 44: 255 60. doi: 10.1016/j.jcrc.2017.10.036.

25.- Londoño J, León A L, Rodríguez F, Barrera L, de la Rosa G, Dennis R, et al. Serum lactate in the emergency department as a prognostic factor in patients with sepsis without hypotension. Med Clin (Barc) 2013; 141 (6): 246-51. doi: 10.1016/j. medcli.2012.05.033.

26.- Shapiro N I, Howell M D, Talmor D, Nathanson L A, Lisbon A, Wolfe R E, et al. Serum lactate as a predictor of mortality in emergency department patients with infection. Ann Emerg Med. 2005; 45 (5): 524-8. doi: 10.1016/j.annemergmed.2004.12.006.

27.- Trzeciak S, Dellinger R P, Chansky M E, Arnold R C, Schorr C, Milcarek B, et al. Serum lactate as a predictor of mortality in patients with infection. Intensive Care Med 2007; 33 (6): 970-7. doi: 10.1007/s00134-007-0563-9.

28.- Vincent J L, de Mendonça A, Cantraine F, Moreno R, Takala J, Suter P M, et al. Use of the SOFA score to assess the incidence of organ dysfunction/failure in intensive care units: results of a multicenter, prospective study. Working group on "sepsis-related problems" of the European Society of Intensive Care Medicine. Crit Care Med 1998; 26 (11): 1793 800. PMID: 9824069. 